\section{Could HIV drugs treat AD?}

Somatic gene recombination of a gene that is central to Alzheimer disease (AD) occurs in the brain, according to a new study published in Nature. The finding could change our understanding of $\mathrm{AD}$ pathogenesis and suggests that existing HIV therapies could be useful in the treatment of AD.

Earlier in his career, Jerold Chun, senior author of the new study, demonstrated that part of the immunological recombinase is expressed in the brain. This finding initiated a search for a gene that undergoes recombination in the brain.

"Finding a gene that was recombined eluded everyone," says Chun, "but many years of research finally pointed to a gene that could be recombined and was of central importance in familial $\mathrm{AD}$ and Down syndrome: $A P P$, the gene that encodes amyloid precursor protein."
Previous work demonstrated that copy number variations of $A P P$ were altered in sporadic $\mathrm{AD}(\mathrm{sAD})$, providing the first indication that the gene could undergo recombination. In the new study, Chun and colleagues analysed APP in detail.

The researchers first analysed APP RNA in small populations of neurons from people with sAD and healthy controls. They identified many previously unseen $A P P$ variants in neurons from patients with $\mathrm{SAD}$ that were rare in neurons from controls. The team then analysed whether neuronal DNA sequences were the source of variants. They used nine distinct approaches, all of which identified genomic complementary DNAs (gencDNAs) that corresponded to the RNA variants identified. The results confirmed the presence of $A P P$ variants in neuronal genomes and showed that the variation arises through genomic mosaicism.
Furthermore, in vitro experiments indicated that the formation of gencDNAs in cells depends on reverse transcriptase activity, presenting a novel potential therapeutic target. Interestingly, Chun and colleagues note that $\mathrm{AD}$ is rare among individuals aged $>65$ years who have received longterm therapy for HIV, which includes reverse transcriptase inhibitors. The combined observations suggest that currently used, FDA-approved HIV therapies, or modifications of these therapies, might be beneficial in the treatment of AD.

"Our findings suggest a new mechanism of neurodegeneration that involves altered or too much recombination producing toxic genes, RNAs and proteins," explains Chun. "We will now work with clinical colleagues to test the hypothesis that appropriate reverse transcriptase therapies can treat AD."

Ian Fyfe

ORIGINAL ARTICLE Lee, M.-H. et al. Somatic APP recombination in Alzheimer's disease and normal neurons. Nature https://doi.org/10.1038/s41586018-0718-6 (2018)

\title{
First genetic risk loci for ADHD identified
}

A genome-wide association study (GWAS) has uncovered the first genetic risk loci for attention deficit hyperactivity disorder (ADHD). The findings shed light on the biological pathways underlying the disorder and might pave the way to new treatments.

ADHD is a neurodevelopmental behavioural disorder characterized by severe impulsivity and inattention. The condition affects $\sim 5 \%$ of children and $2.5 \%$ of adults globally. Previous studies of ADHD have indicated a substantial heritable component of the disorder, but researchers have been unable to identify individual risk loci until now. "It is important to understand the causes and disease mechanisms of ADHD in order to lay the foundation for better treatment of the condition, which is impairing the lives of many millions of people around the world," remarks study author Anders Børglum.

"I realized that solving this problem required many DNA samples from people with and without ADHD," explains study author Stephen Faraone. "So, I created the ADHD Molecular Genetics Network, funded by the US National Institute of Mental Health."

The researchers performed a GWAS in 20,183 individuals diagnosed with ADHD and 35,191 healthy individuals without ADHD. The results revealed 12 risk loci, most of which were associated with genes expressed in the brain, including FOXP2, DUSP6 and SORCS3. Many of the identified variants were in genes involved in brain circuit development and cognitive functions such as learning and language.
Interestingly, the team found that the ADHD-associated variants were linked to traits of impulsivity and inattention in people without $A D H D$ in the general population. ADHD risk alleles were also associated with an increased risk of other traits and disorders, such as depression, type 2 diabetes mellitus, obesity and insomnia.

The loci that the researchers identified only account for a small fraction of the total genetic risk of ADHD. "The vast majority of ADHD genetics is still undiscovered and will require even larger studies," remarks Børglum.

The investigators also note that more work is required to understand the role of the identified genetic loci in ADHD pathogenesis. "Future work will need to map out the biological pathways implicated by our data with the hope that, someday, we will be able to develop treatments to cure or prevent the disorder," concludes Faraone.

Charlotte Ridler

ORIGINAL ARTICLE Demontis, D. et al.

Discovery of the first genome-wide significant risk loci for attention deficit/hyperactivity disorder. Nat. Genet. https://doi.org/10.1038/s41588-0180269-7 (2018) 